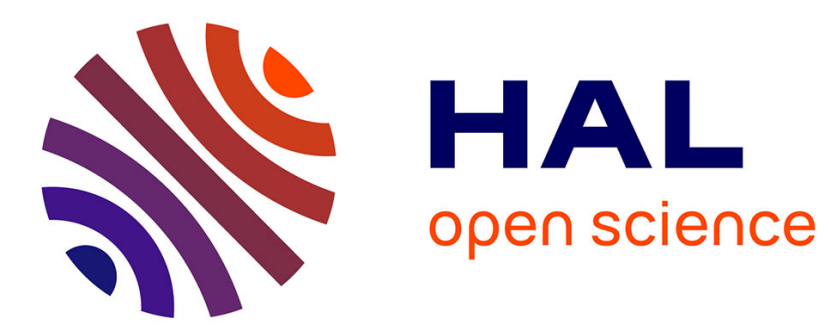

\title{
Slow relaxation mode in concentrated oil-in-water microemulsions consisting of repulsive droplets.
}

Yoshiyuki Hattori, Hideharu Ushiki, Laurent Courbin, Pascal Panizza

\section{To cite this version:}

Yoshiyuki Hattori, Hideharu Ushiki, Laurent Courbin, Pascal Panizza. Slow relaxation mode in concentrated oil-in-water microemulsions consisting of repulsive droplets.. Physical Review E : Statistical, Nonlinear, and Soft Matter Physics, 2007, 75 (2 Pt 1), pp.021504. 10.1103/PHYSREVE.75.021504 . hal-00907961

\section{HAL Id: hal-00907961 \\ https://hal.science/hal-00907961}

Submitted on 22 Nov 2013

HAL is a multi-disciplinary open access archive for the deposit and dissemination of scientific research documents, whether they are published or not. The documents may come from teaching and research institutions in France or abroad, or from public or private research centers.
L'archive ouverte pluridisciplinaire HAL, est destinée au dépôt et à la diffusion de documents scientifiques de niveau recherche, publiés ou non, émanant des établissements d'enseignement et de recherche français ou étrangers, des laboratoires publics ou privés. 


\title{
Slow relaxation mode in concentrated oil-in-water microemulsions consisting of repulsive droplets
}

\author{
Y. Hattori, ${ }^{1}$ H. Ushiki, ${ }^{2}$ L. Courbin, ${ }^{3, *}$ and P. Panizza ${ }^{4, \dagger}$ \\ ${ }^{1}$ Graduate School of Bio-applications and Systems Engineering, Tokyo University of Agriculture and Technology, \\ 2-24-16, Naka-cho, Koganei 184-8588, Japan \\ ${ }^{2}$ Faculty of Agriculture, Tokyo University of Agriculture and Technology, 3-5-8, Saiwai-cho, Fuchu 183-8509, Japan \\ ${ }^{3}$ Division of Engineering and Applied Sciences, Harvard University, Pierce Hall, Cambridge, Massachusetts 02138, USA \\ ${ }^{4}$ Groupe Matière Condensée et Matériaux, UMR-CNRS 6626, Université Rennes 1, 35042 Rennes, France \\ (Received 10 February 2006; revised manuscript received 18 July 2006; published 22 February 2007)
}

\begin{abstract}
The present contribution reports on the observation of two diffusive relaxation modes in a concentrated microemulsion made of repulsive droplets. These two modes can be interpreted in the frame of Weissman's and Pusey's theoretical pioneering works. The fast mode is associated to the collective diffusion of droplets whereas the slow one corresponds to the relaxation of droplet concentration fluctuations associated with composition and/or size. We show that (i) repulsive interactions considerably slow down the latter and (ii) a generalized Stokes Einstein relationship between its coefficient of diffusion and the Newtonian viscosity of the solutions, similar to the Walden's rule for electrolytes, holds for concentrated microemulsion systems made of repulsive droplets.
\end{abstract}

DOI: 10.1103/PhysRevE.75.021504

PACS number(s): 61.20.-p, 66.10.-x, 82.70.-y

\section{INTRODUCTION}

Microemulsions are thermodynamically stable ternary mixtures consisting of water, oil, and surfactants $[1,2]$. The stability of these phases results from a subtle competition between the entropy of mixing and the elasticity of the surfactant film $[3,4]$ separating water from oil domains. If a film is very asymmetric with a strong preferred curvature $c_{0}$, it will preferentially form droplets with radii closed to $c_{0}^{-1}$ [3], whereas if it is symmetric and flexible or weakly asymmetric, its local curvature may strongly fluctuate. This leads to multiply connected networks of oil and water, separated by surfactant monolayers [5-7]. If the static properties of these phases are now well understood, their dynamical properties are by comparison still largely unexplored and remain for some of them so far still controversial.

In the dilute regime, the dynamical behaviors of droplets have been studied mostly using light scattering and spin echo neutron scattering. At low dilutions, light scattering measurements permit to obtain the hydrodynamic size as well as the hydrodynamic interactions between droplets [8], whereas spin echo measurements give information on the droplet shape fluctuations [9]. However, much less work has been spent to study the dynamics of concentred systems made of droplets. To the best of our knowledge, these few studies have mostly been limited to water-in-oil (W/O) systems for which the interactions between droplets are attractive [10]. In such systems, where droplets can be modelled as adhesive quasimonodisperse spheres, percolation and phase separation phenomena are commonly encountered [11-14]. Although, the observations of a long-time diffusive relaxation mode, by means of quasielastic light scattering, has been reported by several groups for attractive droplets [15-17]. The dynamical

\footnotetext{
*Electronic address: lcourbin@deas.harvard.edu

†Electronic address: pascal.panizza@univ-rennes1.fr
}

behavior differs whether the solutions are far from or close to the percolation threshold. Although in both cases, the intensity correlation measured by quasielastic light scattering measurements reveals a fast exponential decay associated to the collective diffusion of droplets, differences are noticeable at long times. For solutions far from the percolation line, the intensity correlation functions present another single exponential decay at longer times. This slow diffusive mode corresponds to the relaxation of droplet concentration fluctuations associated with composition and/or size. It occurs through the self-diffusion of individual droplets $[15,17]$. Comparatively, solutions close to the percolation line show no longer a single exponential but instead a stretched exponential decay at long times [18]. This behavior is characteristic of strongly interacting systems with many degrees of freedom, involving different relaxation channels [19]. Indeed, the slow diffusive mode is herein related to the existence of polydispersed fractal clusters.

In this paper, we study the dynamic properties of a concentrated system made of oil-in-water $(\mathrm{O} / \mathrm{W})$ droplets. Contrary to W/O microemulsions, O/W droplets can be charged [20] and therefore present repulsive interactions leading eventually to the formation of long range ordered structures [21-23]. Our present work investigates the influence of interactions on the slow dynamical properties of microemulsion systems. Using dynamic light scattering (DLS) experiments [24], we report the existence of two diffusing modes. We show that the slow relaxation mode is strongly correlated to the viscosity of the solution, since a generalized Stokes Einstein relationship holds, provided that the viscosity of the solvent is replaced by that of the solution.

\section{EXPERIMENTAL}

\section{A. Materials}

We study a pseudoquaternary system composed of brine, paraffin oil (Marcol 52, purchased from ESSO, Inc.), non- 
ionic (NIS) and anionic (AS) surfactants. For NIS and AS surfactants we use SimsolP4 and Sulfosuccinate (AOT), respectively, purchased from Seppic and Fluka (France). Simsol P4 is a commercial non ionic surfactant containing mainly $\mathrm{C}_{12}(\mathrm{EO})_{4}$. We study the phase diagram of this system for solutions where the respective weight ratios of AOT over Marcol oil and AOT over Simsol P4 are $W=$ AOT/Marcol $=1 / 3$ and $X=$ AOT $/$ Simsol $P 4=1 / 2$. At $25^{\circ} \mathrm{C}$, the densities of AOT, Simsol P4 and Marcol 52 are, respectively, 1.13, 0.901 , and $0.815 \mathrm{~g} / \mathrm{cm}^{3}$. Solutions are prepared as follows. Marcol 52 and Simsol P4 are first mixed together and then added to AOT. The resulting mixture is heated at $70{ }^{\circ} \mathrm{C}$ for a few minutes upon total dilution of AOT. Brine, prepared with Millipore water, is then added to our mixture and the obtained solution is left at rest for a few days.

\section{Experiments and setup}

For each experiment, the experimental cell is connected to a water bath allowing us to control the sample temperature to within $0.1^{\circ} \mathrm{C}$ [dynamic light scattering (DLS), viscosity, and conductivity measurements] or $0.01{ }^{\circ} \mathrm{C}$ [small angle $\mathrm{x}$ ray scattering (SAXS) experiments].

DLS experiments are performed using a linear polarized Argon laser operating in air at $\lambda=488 \mathrm{~nm}$ and a temperature controlled cylindrical scattering cell. The scattered light is collected with a photon-counting photomultiplier tube for scattering angles $\theta$, at $60^{\circ}, 70^{\circ}, 90^{\circ}, 110^{\circ}, 120^{\circ}$ and $135^{\circ}$. Time correlation functions of the scattered light intensity $g_{2}(q, t)=\langle I(q, t) I(q, 0)\rangle$ are accumulated in homodyne detection using a 288 channels multiple tau autocorrelator (ALV5000, Germany). The time autocorrelation function of the scattered light intensity can be related to the autocorrelation function of the electric field fluctuations $g_{1}(q, t)$ through

$$
g_{2}(q, t)=\left[1+\gamma\left|g_{1}(q, t)\right|^{2}\right],
$$

where $\gamma$ which is the coherence factor of the experiment should ideally be equal to 1 . However, because of the loss of coherence inherent to any experimental setup and due to possible multiple scattering, $\gamma$ takes smaller values. In our experiments, the coherence factor is proven to be better than 0.9 . We recall that $q=(4 \pi n / \lambda) \sin (\theta / 2)$, where $n$ is the optical index of refraction of the scattering medium and $\theta$ is the scattering angle.

Viscosity measurements are made with a commercial stress controlled rheometer (TA instruments AR1000, USA) using a Couette cell geometry. The rotor and stator radii of this cell are, respectively, 24 and $25 \mathrm{~mm}$, while its height is $40 \mathrm{~mm}$.

The conductivity and dielectric constants are measured as a function of the applied voltage frequency with a cylindrical cell using an impedance analyzer 4192A LF (Hewlett Packard) and a data acquisition board (National Instrument). With this setup, the frequency can vary between $5 \mathrm{~Hz}$ and $13 \mathrm{MHz}$.

SAXS experiments are performed on the ID2 beam line, at the European Synchroton Facilities (ESRF) in Grenoble, France. The wavelength of the incident monochromatic radiation and the beam size were, respectively, $0.1 \mathrm{~nm}$ and

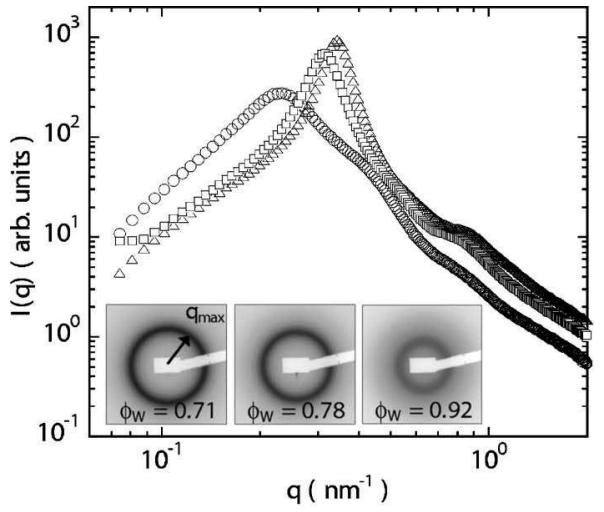

(a)

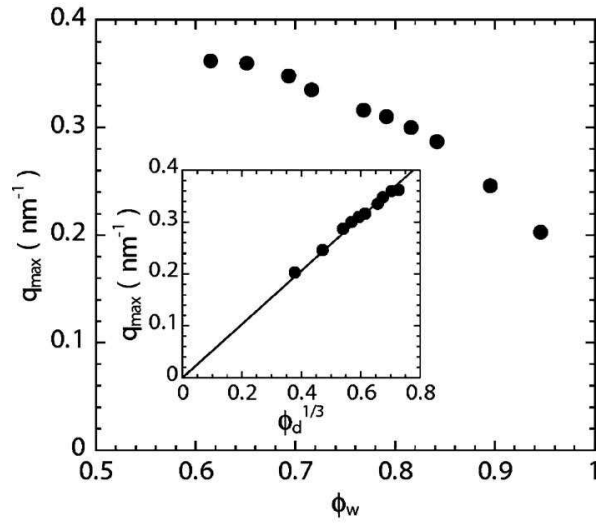

(b)

FIG. 1. (a) SAXS intensity $I(q)$ for different water volume content $\phi_{W}:(\triangle) \phi_{W}=0.71,(\square) \phi_{W}=0.78$, and $(\bigcirc) \phi_{W}=0.92$. Inset: SAXS patterns corresponding to the spectra presented in (a). (b) Evolution of $q_{\max }$, the position of the Bragg peak observed in SAXS as a function of $\phi_{W}$. Inset: variation of $q_{\max }$ with $\phi_{d}^{1 / 3}$, where $\phi_{d}$ is the total volume fraction of the oil and both surfactants. The continuous line is the best linear fit $q_{\max }\left(\mathrm{nm}^{-1}\right)=0.516 \phi_{d}^{1 / 3}$.

$100 \times 300 \mu \mathrm{m}^{2}$. The scattered intensity is collected on a two dimensional detector placed $3 \mathrm{~m}$ away from the sample and recorded by an image intensified charge-coupled device detector with an active area of size about $230 \times 230 \mathrm{~mm}^{2}$ and a square pixel size $\sim 0.175 \mathrm{~mm}$.

\section{RESULTS}

\section{A. Structure}

Figure 1(a) shows the evolution of the SAXS patterns observed along a dilution line at $T=25^{\circ} \mathrm{C}$ for water volume content $\phi_{w}$ ranging from 0.71 up to 0.92 . The presence of a peak at $q=q_{\max }$ reveals the existence in the solutions of a well defined correlation length $d=2 \pi / q_{\max }$ which corresponds to the mean distance between dispersed objects. As shown in Fig. 1(b), this distance increases with the water volume fraction.

Both this variation and the high value of the measured zero frequency conductivity $\sigma$ [see Fig. 2(a)] prove that the system is of $\mathrm{O} / \mathrm{W}$ type. Along the dilution line $(W=1 / 3, X$ $=1 / 2$ ), let us therefore call $\phi_{d}$ the volume fraction of the dispersed phase made of oil, AOT and Simsol P4. As shown 


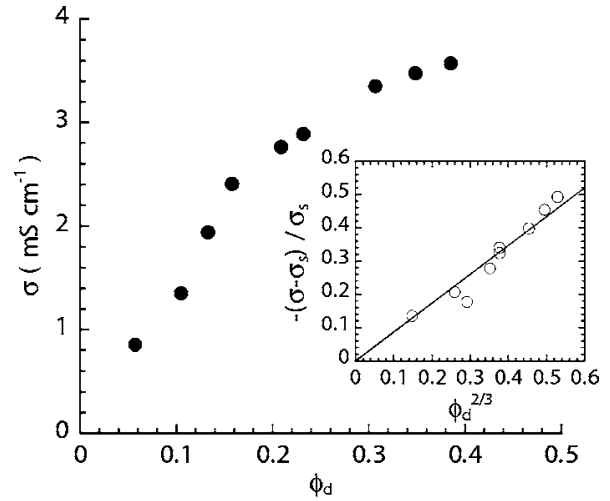

(a)

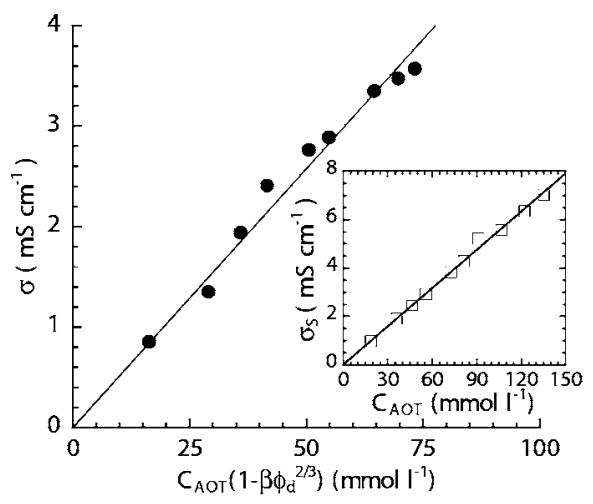

(b)

FIG. 2. (a) Variation of $\sigma$, the low frequency conductivity as a function of $\phi_{d}$. Inset: shown is $-\left(\sigma-\sigma_{s}\right) / \sigma_{s}$ as a function of $\phi_{d}^{2 / 3}$. The continuous line which corresponds to the best linear fit has slope $\beta \sim 1$. (b) Evolution of $\sigma$ vs $c_{\mathrm{AOT}}\left(1-\beta \phi_{d}^{2 / 3}\right)$, where $\beta$ is the numerical constant one (a). Inset: shown is the variations of $\sigma_{s}$ as a function of $c_{\text {АОт }}$. Both solid lines stand for the best linear fits which value of the slope is $51.5 \mathrm{~S} \mathrm{~cm}^{2} / \mathrm{mol}$.

in Fig. 1, the variation of $q_{\max }$ with $\phi_{d}$ follows a power law $q_{\max } \sim \phi_{d}^{\alpha}$. The value $1 / 3$ found for $\alpha$ rules out the possibility that the structure of the microemulsion may consist of two dimensional membranes $(\alpha=1)$ or long cylinders $(\alpha=1 / 2)$. It instead proves that the microemulsion is made of quasimonodisperse globular $\mathrm{O} / \mathrm{W}$ objects $\left(d \sim \phi_{d}^{-1 / 3}\right)$. Another experimental fact in favor of this is obtained with conductivity measurements.

As shown in the inset of the Fig. 2(a), the variation of $\sigma$, the zero frequency conductivity, with $\phi_{d}$ is well accounted by the obstruction factor of monodisperse spheres $\sigma\left(\phi_{d}\right)$ $=\sigma_{s}\left(1-\beta \phi_{d}^{2 / 3}\right)$, where $\beta \sim 1$ is a numerical constant characteristic of the packing of the spheres and where $\sigma_{s}$ is the conductivity of the solvent. The electrical transport occurs because of the presence in the water solvent of cationic $\mathrm{Na}^{+}$ ions; $\sigma_{s}$ should therefore be proportional to $c_{\mathrm{Na}^{+}}$their concentration in solution. Assuming that all AOT molecules are ionized, we obtain $c_{\mathrm{Na}^{+}} \sim c_{\mathrm{AOT}}$, where $c_{\mathrm{AOT}}$ is the molar concentration of AOT molecules per $\mathrm{cm}^{3}$. To check the validity of this assumption, we plot $\sigma\left(\phi_{d}\right)$ as a function of $c_{\text {AOT }}\left(1-\beta \phi_{d}^{2 / 3}\right)$. As shown in Fig. 2(b) and in its inset, an excellent agreement is found. The slope of the best linear fit has units of $\mathrm{S} \mathrm{cm}^{2} / \mathrm{mol}$ and its value is very close to

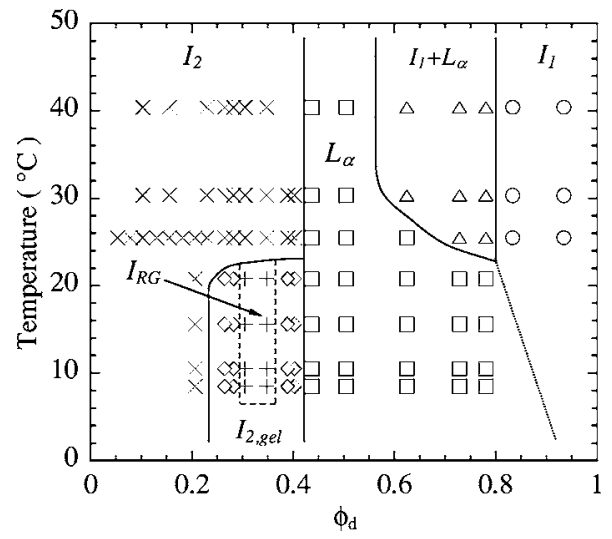

FIG. 3. Phase diagram of the system for $X=1 / 2$ and $W=1 / 3$ as a function of $\phi_{d}$ and the temperature. $I_{1}$ and $I_{2}$, stand, respectively, for the $\mathrm{W} / \mathrm{O}$ and $\mathrm{O} / \mathrm{W}$ droplet microemulsion phase. $I_{2}$, gel, and $I_{\mathrm{RG}}$ correspond to the regions where the $\mathrm{O} / \mathrm{W}$ droplet phase behaves as an elastic solid and as a so-called "ringing gel." $L_{\alpha}$ stands for the lamellar phase region.

$50.1 \mathrm{~S} \mathrm{~cm}^{2} / \mathrm{mol}$, the ion conductivity of $\mathrm{Na}^{+}$. Our experiments show that the microemulsion system consists of $\mathrm{O} / \mathrm{W}$ droplets; due to the presence of ionized AOT in the solvent these droplets are charged [20].

Figure 3 shows the phase diagram of the system as a function of $\phi_{d}$ and the temperature. As previously discussed, for $0<\phi_{d}<0.4$, the system is a $\mathrm{O} / \mathrm{W}$ droplet microemulsion phase (region $I_{2}$ ). When $\phi_{d}$ increases, the isotropic solutions become more and more viscous. At lower temperatures, for $0.3<\phi_{d}<0.35$, the solutions exhibit a so-called "ringing gel" behavior (see the $I_{\mathrm{RG}}$ region in Fig. 3). In this temperature region of the phase diagram, snapping the container with the solution inside leads to a "ringing" mechanical response similar to that of metals [25]. In this region, the droplets present a long range order [26]. For $0.4<\phi_{d}<0.78$, the system exhibits a lamellar structure $\left(L_{\alpha}\right)$, well evidenced by both the presence of Bragg peaks in SAXS spectra and birefringence. For $\phi_{d}>0.78$, the solutions are transparent and low viscous, exhibit no birefringence at rest or under flow and present a very low conductivity indicating that the structure is now of W/O type (region $I_{1}$ ).

Now that the structure is well characterized, let us turn to the dynamic properties of the $\mathrm{O} / \mathrm{W}$ microemulsion in the $I_{2}$ region of the phase diagram (i.e., for $0<\phi_{d}<0.4$ at $T=25^{\circ} \mathrm{C}$ ).

\section{B. Dynamic properties}

Figure 4 shows the normalized time autocorrelation functions of the electrical field at an angle of $90^{\circ}$ for different values of $\phi_{d}$ in the $I_{2}$ region of the phase diagram. As evident, our systems are ergodic and therefore at equilibrium since for each graphs, $\lim _{t \rightarrow \infty} g_{1}(t)=0$.

To analyze the two relaxation modes easily distinguishable when $\phi_{d}>0.1$, we first fit our data with the sum of a two exponential functions. For $\phi_{d} \leqslant 0.1$, it is difficult to resolve two exponentials unambiguously, and in this case one apparent relaxation mode is observed [see the curve obtained 

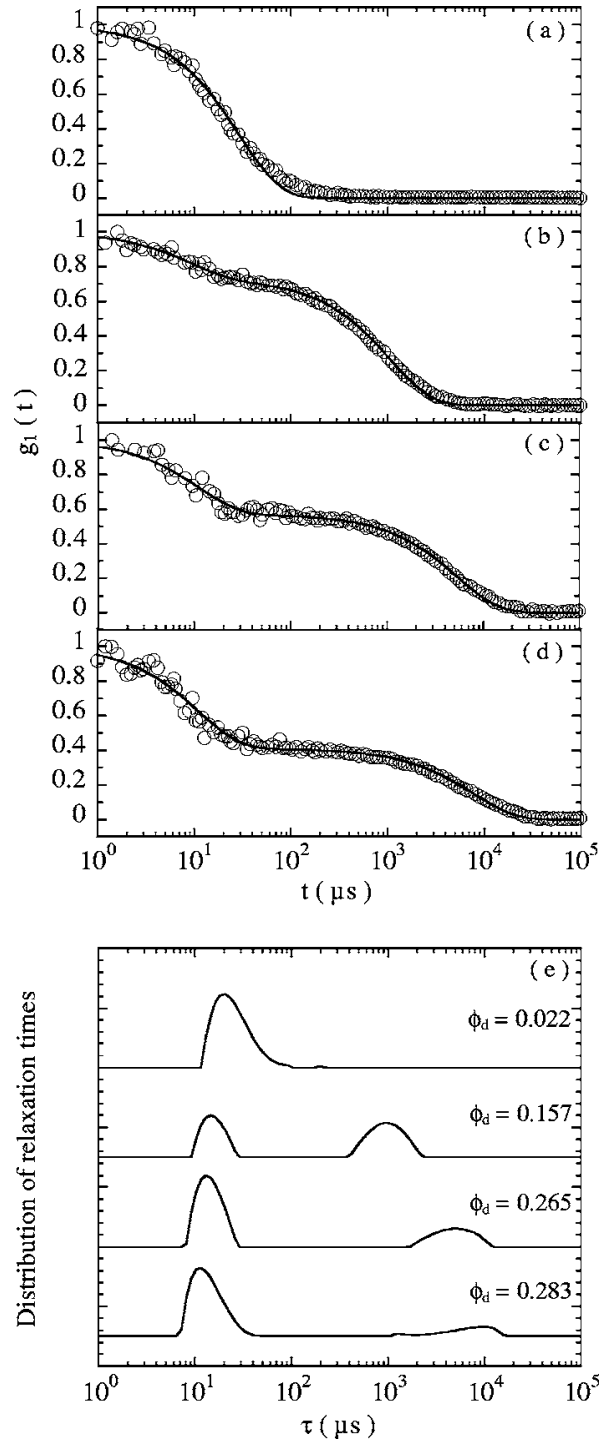

FIG. 4. Normalized autocorrelation functions obtained at an angle of $90^{\circ}$ for (a) $\phi_{d}=0.022$, (b) 0.157 , (c) 0.265 , and (d) 0.283 . For (b), (c), and (d) the continuous lines are the best fits obtained for the data with the sum of two exponentials (b) $g_{1}(t)$ $=0.27 \exp (-t / 9)+0.73 \exp (-t / 1057), \quad(\mathrm{c}) \quad g_{1}(t)=0.43 \exp (-t / 11)$ $+0.57 \exp (-t / 5358), \quad$ and $\quad(\mathrm{d}) \quad g_{1}(t)=0.59 \exp (-t / 11)$ $+0.41 \exp (-t / 7680)$. For (a) The continuous line is the best fit obtained with a monoexponential, namely, $g_{1}=\exp (-t / 29)$, where $t$ is the given in $\mu \mathrm{s}$. The temperature is $T=25{ }^{\circ} \mathrm{C}$. (e) Distribution of the relaxation times obtained by an inverse Laplace transformation of the autocorrelation functions presented in Figs. 4(a)-4(d).

for $\phi_{d}=0.22$ in Fig. 4(a)]. Moreover, the DLS measurements performed in this very dilute region of the phase diagram give us another proof in favor of a droplet structure. The value of the diffusion coefficient $D_{0}$ obtained for $\phi_{d}=0.022$ is equal to $D_{0}=3.00 \times 10^{-11} \mathrm{~m}^{2} \mathrm{~s}^{-1}$. At such low dilution, this coefficient roughly corresponds to the self-diffusion coefficient of an isolated droplet. Using the Stokes-Einstein relationship, the hydrodynamic radius of droplets can therefore be obtained by $R_{h}=k_{B} T / 6 \pi \eta_{s} D$, where $\eta_{s}$ and $k_{B} T$, respectively, stand for the viscosity of the solvent and the thermal energy. The value, we found, $R_{h} \sim 7.3 \mathrm{~nm}$, is quite consistent

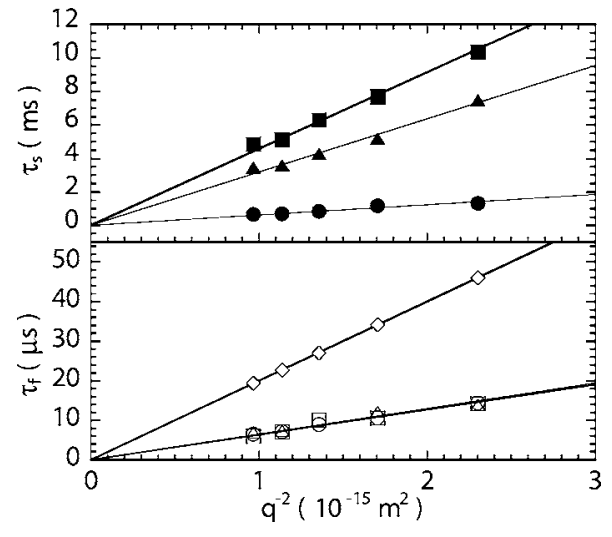

FIG. 5. Variation of both slow ( $\tau_{s}$, closed symbols) and fast $\left(\tau_{f}\right.$, open symbols) characteristic times as a function of $q^{-2}$ for $\phi_{d}$ $=0.022(\diamond), 0.157(\bullet, \bigcirc), 0.265(\boldsymbol{\Delta}, \triangle)$, and $0.283(\boldsymbol{\square}, \square)$.

with an estimation of the droplet radius inferred from the structure analysis with SAXS [26].

As shown in Fig. 4, the analysis of the two relaxation modes via the fitting of our results with a sum of two exponential functions is in relatively good agreement with our experimental findings, indicating the existence of two well defined characteristic times in the system. Also, we analyzed the DLS results presented in Fig. 4 with an inverse Laplace transform method [see Fig. 4(e)]. As shown in this figure, the inverse Laplace method clearly demonstrates the existence of two characteristic times in the microemulsion system. For simplicity's sake, further in the article we analyze our DLS measurements with a sum of two exponential functions as it is usually done for this kind of study [27]. The characteristic time $\tau_{s}$ associated to slow relaxation mode, strongly increases with $\phi_{d}$, whereas the characteristic time $\tau_{f}$ associated to the fast relaxation mode, remains quite constant. As depicted in Fig. 5, both modes are diffusive since $\tau_{f}$ and $\tau_{s}$, vary linearly with $q^{-2}$.

The values of the two diffusion coefficients associated to both diffusing modes can be measured from the respective inverse slopes of the best linear fits $\tau_{f}$ and $\tau_{s}$ versus $q^{-2} . D_{f}$, the diffusion coefficient associated to the fastest mode increases slightly with $\phi_{d}$ (Fig. 6). In contrast, the decrease of the slow mode diffusion coefficient $\left(D_{s}\right)$ with $\phi_{d}$ is extremely strong. When $\phi_{d} \rightarrow 0$, both diffusion coefficients tend towards the same value: $D_{0} \sim 3.0 \times 10^{-11} \mathrm{~m}^{2} \mathrm{~s}^{-1}$, corresponding to the self-diffusion coefficient of one droplet and leading to the following value for the hydrodynamic radius of droplets $R_{h} \sim 7.3 \mathrm{~nm}$.

\section{DISCUSSION}

The existence of two diffusive modes has already been reported for concentrated microemulsion systems exhibiting a bicontinuous structure [27] or consisting of attractive droplets $[15,17]$, as well as for other systems such as protein dispersions [28] and charged colloidal silica or latex suspensions [29,30]. A possible origin of the existence of such a phenomenon in concentrated systems has been proposed by Weissman [29] and Pusey et al. [31], some times ago. Ac- 


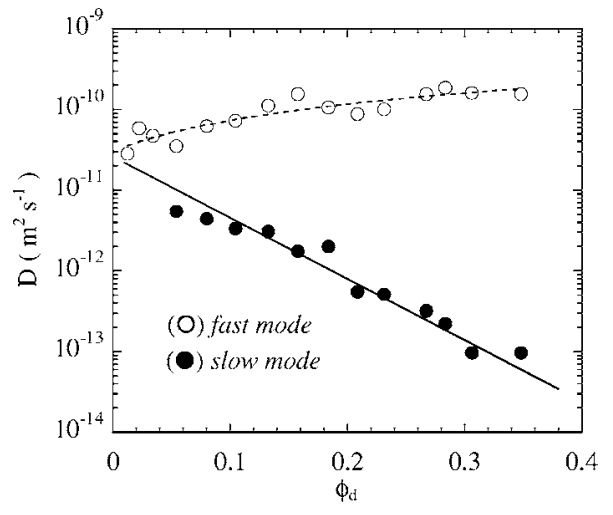

FIG. 6. Variation of the fast $\left(D_{f}, \bigcirc\right)$ and slow $\left(D_{s}, 0\right)$ diffusion coefficients obtained by DLS measurements with $\phi_{d}$. The dashed line represents the best linear fit of $D_{s}$, namely, $D_{s}=3.0 \times 10^{-11}\left(1+14 \phi_{d}\right)$. The continuous line corresponds to the best exponential fit.

cording to these authors, concentrated colloidal dispersions with a slight size or optical index polydispersity, may present two distinct diffusion modes in DLS measurements. The fastest mode is associated to the collective diffusion and the slow mode to the self-diffusion of particles. The collective diffusion relaxes a fluctuation of $N$, the local number density of droplets regardless of their scattering power. In such relaxation process, the distance between particles varies whereas their initial spatial arrangement remains identical, i.e., they keep the same nearest neighbors. The diffusion coefficient associated to this process is therefore directly related to $\Pi$, the osmotic pressure of the solution. It is given by the following relationship: $D_{f}=f \partial \Pi / \partial N$, where $f$ stands for the particle mobility. For dilute solutions where the particles interact via an overall repulsive potential, $D_{f}$ increases weakly with $\phi_{d}$ and can be written as $D_{f}=D_{0}\left(1+\alpha \phi_{d}\right)$, where $D_{0}$ is the infinite dilution value of the collective diffusion coefficient and $\alpha$ is the first dynamical virial coefficient of $D_{f}\left(\alpha=1.45\right.$ for hard spheres [32]). In the $\phi_{d} \rightarrow 0$ limit, one retrieves the Stokes-Einstein formula for a single particle $D_{0}=k_{B} T / 6 \pi \eta_{s} R_{h}$. These predictions are in agreement with our observations for the fast mode.

Within this framework, once the number density of particles has relaxed to a homogeneous value, fluctuations of polarization may still persist because of local fluctuations of the relative proportion of the particles with different scattering power. In colloidal suspensions, such fluctuations can only relax through the self-diffusion of the different particles. Since, in the $\phi_{d} \rightarrow 0$ limit, the value of the self-diffusion coefficient is set by the Stokes-Einstein formula, the diffusion coefficient associated with the fast mode (collective diffusion) and the slow modes (self-diffusion) are identical. This therefore explains the absence of two modes at low particle concentrations. Hence, the two diffusing modes can only be observed for high concentration when the interaction between particles becomes sufficiently large so that the two diffusion coefficients become distinguishable. The variation of the two diffusion coefficients with volume fraction is different. The self-diffusion coefficient describing polydispersity fluctuations should decrease strongly with $\phi_{d}$. Such a variation is easy to understand qualitatively. When a Brownian particle moves through a concentrated dispersion for some time, it feels on average a net friction force arising not only from the solvent but also from its interactions with other particles which strongly slow down its motion. These predictions given by Weissman and Pusey seem therefore in good qualitative agreement with our experimental findings for the slow mode.

For microemulsion systems, size polydispersity of droplets always exists due to thermal fluctuations [33]. For a AOT/water/heptane system, Kotlarchyk et al. [34] have observed size polydispersity of $30 \%$ in agreement with theoretical predictions [33]. In our system, since the surfaces of droplets are covered by a combination of an anionic and an non-ionic surfactants, fluctuations of the composition of the droplet surface and therefore of its charge may also exist [35-37]. Hence, microemulsion systems differ therefore drastically from colloidal suspensions since the droplets can deform and eventually exchange matter with each other. Consequently, a possibility to equilibrate size or composition fluctuation in these systems, which does not exist for colloidal suspension, is through spontaneously thermally activated exchange of the oil or ionic surfactant molecules between dispersed domains [38]. This process which involves the permeation of oil or surfactant from one droplet to another one should be energy activated since it involves the fusion of domains which requires the creation and destruction of necks.

Let us assume that the droplet charges and sizes remain frozen during the duration of our DLS experiments. The mechanism which can equilibrate droplet concentration fluctuations associated with charge and size is then the relative diffusion of droplets with different sizes and charges. Under this assumption, it then follows that the characteristic relaxation time associated to self-diffusion given in our experiments by $\tau \sim D q^{2} \sim 10^{-5} \mathrm{~s}$ is much larger than $\tau_{p}$ the characteristic time of permeation between droplets. Since an estimation of $\tau_{c}$, the collision time between two adjacent droplets is roughly given by $\tau_{c} \sim D_{0}(d-2 R)^{2} \sim 10^{-8} \mathrm{~s}$, it follows that the probability that two colliding droplets exchange matter is less than 1/1000, a result in good agreement with a previous result [12] which validates our assumption.

Since the slow mode is related to the self diffusion of droplets, the simplest picture to describe it is to consider that the droplet is in a cage. In this cage it behaves as an harmonically bound Brownian oscillator whereas the droplet diffuses over long time out of the cage with a diffusion constant $D_{s}[39,40]$. Within this picture, the slow mode, we observe, requires the escape of the droplet from its cage. The activation energy associated to this process should therefore strongly increase with $\phi_{d}$ since the mean distance between adjacent droplets roughly varies as $\phi_{d}^{-1 / 3}$. This is exactly what we observe experimentally (see Fig. 7). Predicting the variation of $D_{s}\left(\phi_{d}\right)$ is a very challenging task, because of the central role of hydrodynamic interactions in this process. For hard spheres, an analytical calculation leads in the first order in volume fraction to $D_{s}\left(\phi_{d}\right)=D_{0}\left(1-2.1 \phi_{d}\right)[41,42]$. For higher droplet volume fractions, theoretical works either ne- 


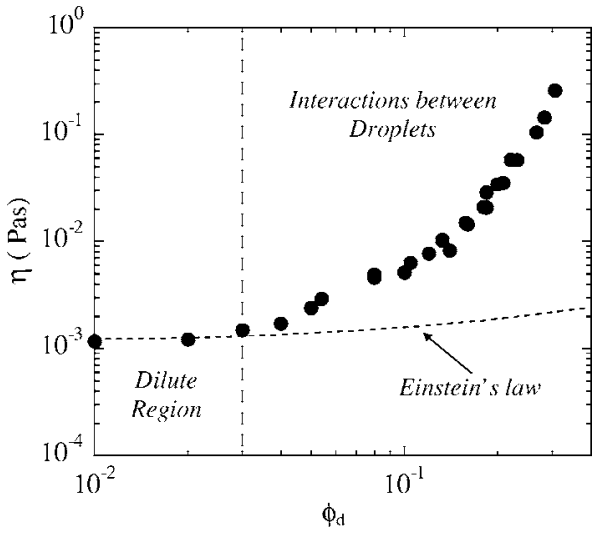

FIG. 7. Viscosity of the solutions $(-)$ versus $\phi_{d}$. The dashed line corresponds to the expected viscosity using Einstein's law $\eta$ $=\eta_{s}\left(1+2.5 \phi_{d}^{\text {eff }}\right)$ with $\phi_{d}^{\text {eff }}$ the effective volume fraction of the charged droplets. The values of $\phi_{d}^{\text {eff }}$ are obtained by using the relation $\phi_{d}^{\text {eff }}=\phi_{d}(1+l / R)^{3}$, where $l$ is the Debye length computed for the solution with $\phi_{d}$.

glect hydrodynamic interactions [43] or consist of Brownian numerical simulations [44]. For our system, we witness a very important variation of $D_{s}\left(\phi_{d}\right)$ with $\phi_{d}$, since the value of $D_{s}$ decreases by more than two decades when $\phi_{d}$ varies only from 0 to 0.4 . This decrease is much stronger than the ones reported for the same range of volume fractions, by other authors on attractive W/O droplets systems. For instance, on systems made of AOT, water and p-Xylene [15] or of AOT, water and alcane [16,17], $D_{s}$ decreases only by one decade. With no doubt, such a difference is related to the nature of the interactions between droplets, attractive for W/O droplets and repulsive in our case. For charged spheres, $D_{s}\left(\phi_{d}\right)$ should exhibit a very strong decrease as $\phi_{d}$ increases, since the repulsive interaction between particles makes it very difficult for a particle to escape its cage. Recall that a rigid particle can escape its cage only if the distance between two of its adjacent droplets increases transiently by more than one droplet diameter and a hole opens up. For microemulsion systems, shape deformation could occur when a particle escapes its confining cage. As a consequence, such a thermally activated process would not require in principle a hole larger than a droplet diameter. For attractive systems, such as for instance Baxter spheres, such a process is more favorable since attraction between neighboring droplets favors links between two droplets and therefore enhance density fluctuations in the neighboring crown, and consequently the opening up of holes [45]. Experimentally, this is what is witnessed. Thus, the decrease of $D_{s}$ with $\phi_{d}$ reported on charged silica spheres [46] is of the same order as the one we observe on our repulsive microemulsion droplets.

Let us now turn to another interesting point. For hard spheres and micelles a remarkable similarity between the slow diffusion coefficient $D_{s}\left(\phi_{d}\right)$ and the inverse of the low shear viscosity of the dispersion $\eta\left(\phi_{d}, \dot{\gamma} \rightarrow 0\right)$ has been noted. Physically the following interpretation can be given. When a Brownian droplet is moving through the concentrated dispersion for some time, it feels on average a friction force arising not only from the solvent but also from the

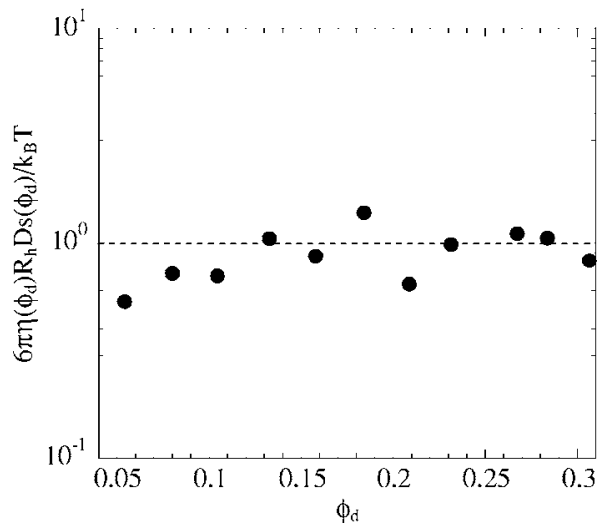

FIG. 8. Variation of $6 \pi \eta\left(\phi_{d}\right) R_{h} D_{s}\left(\phi_{d}\right) / k_{B} T$ as a function of $\phi_{d}$.

interactions with other droplets as well. Assuming that the total friction force obeys the Stokes equation with the solvent viscosity, $\eta_{s}$ replaced by the dispersion low shear viscosity $\eta\left(\phi_{d}, \dot{\gamma} \rightarrow 0\right)$ leads to the following relationship:

$$
D_{s}\left(\phi_{d}\right)=\frac{k_{B} T}{6 \pi \eta\left(\phi_{d}, \dot{\gamma} \rightarrow 0\right) R_{h}} .
$$

As experimentally shown, such a relation is valid for both atomic and molecular liquids, although the factor of 6 must be replaced by a factor of about 3 to yield an accurate agreement [47]. For electrolytes, a similar relation, known in Literature as the Walden rule still holds. Such a remarkable similarity between $D_{s}$ and viscosity has even been observed for hard spheres [48] and micelles [49]. Large theoretical efforts have been undertaken to derive such an empirical relation for atomic fluids and colloidal dispersions [50]. More recently, by using a generalized Einstein's relationship Imhof et al. [46], have shown that the strong decrease of $D_{s}\left(\phi_{d}\right)$ with $\phi_{d}$ observed on charge stabilized colloidal spheres is correlated to the low shear viscosity of the solutions, using a generalized Einstein's relationship. In order to check whether such a correlation still exists between $D_{s}$ and viscosity for concentrated droplet microemulsions, we have measured the viscosity, $\eta$, along the dilution line $(W=1 / 3$, $X=1 / 2)$ and compare its variation with $\phi_{d}$ to that of $D_{s}\left(\phi_{d}\right)$ along the same dilution line.

Figure 7 shows the variation of $\eta$ with $\phi_{d}$. When $\phi_{d}$ is lower than 0.03 , a good agreement is obtained between the measured viscosity and Einstein's law, whereas above this value, deviations become important due to electrostatic and hydrodynamic interactions between droplets. As clearly shown in Fig. 8, there is a strong correlation between both physical quantities.

To conclude, a generalized Stokes-Einstein relationship seems valid for concentrated droplet microemulsion systems provided that the viscosity of the solvent is replaced by that of the solution. We believe that the occurrence of such relationships in many different systems calls for a clarification on theoretical grounds. 


\section{ACKNOWLEDGMENTS}

Y. Hattori acknowledges the financial support of the French-Japanese exchange program. The authors wish to thank T. Narayanan for technical assistance at ESRF and fruitful discussions. We thank T. Bickel and A. Wurger for helpful discussions. Y. Amarouchene, A. Macary, and F. Darnat are acknowledged for their help with viscosity measurements.
[1] T. P. Hoar and J. H. Schulman, Nature (London) 102, 152 (1943).

[2] See for instance, Micelles, Membranes, Microemulsions and Monolayers, edited by W. M. Gelbart, A. Ben-Shaul, and D. Roux (Springer Verlag, New York, 1994).

[3] W. Helfrich, Z. Naturforsch. C 28, 693 (1973).

[4] P. G. de Gennes and C. Taupin, J. Phys. Chem. 86, 2294 (1982).

[5] L. E. Scriven, Nature (London) 63, 123 (1976).

[6] G. Cristobal, J. Rouch, A. Colin, and P. Panizza, Phys. Rev. E 62, 3871 (2000).

[7] P. Panizza, L. Courbin, G. Cristobal, J. Rouch, and T. Narayanan, Physica A 322, 38 (2003).

[8] D. Langevin, Acc. Chem. Res. 21, 255 (1988).

[9] T. Hellweg, M. Gradzielski, B. Farago, and D. Langevin Colloids Surf., A 183-185, 159 (2001).

[10] B. Lemaire, P. Bothorel, and D. Roux J. Phys. Chem. 87, 1023 (1983).

[11] S. Bhattacharya, J. P. Stokes, M. W. Kim, and J. S. Huang, Phys. Rev. Lett. 55, 1884 (1985).

[12] P. Fletcher, A. M. Howe, and B. H. Robinson, J. Chem. Soc., Faraday Trans. 1 83, 985 (1987).

[13] L. Letamendia, E. Pru-Lestret, P. Panizza, J. Rouch, F. Sciortino, P. Tartaglia, C. Hashimoto, H. Ushiki, and D. Risso, Physica A 300, 53 (2001).

[14] Y. Hattori, H. Ushiki, W. Engl, L. Courbin, and P. Panizza, Physica A 353, 29 (2005).

[15] J. H. R. Clarke, J. D. Nicholson, and K. N. Regan, J. Chem. Soc., Faraday Trans. 1 81, 1173 (1985).

[16] Y. D. Yang and J. H. R. Clarke, J. Chem. Phys. 93, 4501 (1990).

[17] A. Shukla, H. Graener, and R. H. H. Neubert, Langmuir 20, 8526 (2004).

[18] J. Rouch, P. Tartaglia, and S. H. Chen, Phys. Rev. Lett. 71, 1947 (1993).

[19] R. G. Palmer, D. L. Stein, E. Abrahams, and P. W. Anderson, Phys. Rev. Lett. 53, 958 (1984).

[20] B. Farago and M. Gradielski, J. Chem. Phys. 114, 10105 (2001).

[21] M. Gradzielski and H. Hoffman, Adv. Colloid Interface Sci. 42, 149 (1992).

[22] M. Gradzielski and H. Hoffman, J. Phys. Chem. 98, 2613 (1994).

[23] P. D. I. Fletcher and R. Johannson, J. Chem. Soc., Faraday Trans. 90, 3567 (1994).

[24] Dynamics Light Scattering, edited by B. J. Berne and R. Pecora (Wiley, New-York, 1976).

[25] G. Oetter and H. Hoffmann, Colloids Surf. 38, 225 (1989).

[26] From the conservation of the dispersed volume fraction, it is possible to derive an expression between the radius of the monodisperse droplets $R$ and $d=2 \pi / q_{\max }$, the mean distance between droplet obtained by SAXS measurement. Assuming a centered cubic structure, for which $R=d\left(\phi_{\mathrm{d}} / \sqrt{3} \pi\right)^{1 / 3}$, and using the best fit of the SAXS measurements (see Fig. 1), one obtains $R \approx 6.9 \mathrm{~nm}$. In the so-called ringing gel regions, SAXS spectra presents several peaks characteristic of a long range order (unpublished results).

[27] U. Peter, D. Roux, and A. K. Sood, Phys. Rev. Lett. 86, 3340 (2001).

[28] P. Licinio and M. Delaye, J. Colloid Interface Sci. 132, 1 (1989).

[29] M. Weissman, J. Chem. Phys. 72, 231 (1980).

[30] P. S. Dalberg, A. Boe, K. A. Strand, and T. Sikkeland, J. Chem. Phys. 69, 5473 (1978).

[31] P. N. Pusey, H. M. Fijnaut, and A. Vrij, J. Chem. Phys. 77, 4270 (1982); P. N. Pusey and R. J. A. Tough, Adv. Colloid Interface Sci. 16, 143 (1982).

[32] G. K. Batchelor, J. Fluid Mech. 52, 245 (1972); 74, 1 (1976).

[33] S. A. Safran, J. Chem. Phys. 78, 2073 (1983).

[34] M. Kotlarchyk, S. H. Chen, and J. S. Huang, J. Phys. Chem. 86, 3273 (1982); 79, 2461 (1983).

[35] H. F. Eicke, M. Borkovec, and B. Das-Gupta, J. Phys. Chem. 93, 314 (1989).

[36] D. G. Hall, J. Phys. Chem. 94, 429 (1990).

[37] J. Appell, G. Porte, and E. Buhler, J. Phys. Chem. B 109, 13186 (2005).

[38] S. T. Milner, M. E. Cates, and D. Roux, J. Phys. (Paris) 51, 2629 (1990).

[39] R. Piazza and V. Degiorgio, Phys. Rev. Lett. 67, 3868 (1991).

[40] J. A. Weiss, A. E. Larsen, and D. G. Grier, J. Chem. Phys. 109, 8659 (1998).

[41] G. K. Batchelor, J. Fluid Mech. 131, 155 (1983).

[42] B. Cichocki and B. U. Felderhof, J. Chem. Phys. 89, 3705 (1988).

[43] J. A. Leegwater and G. Szamel, Phys. Rev. A 46, 4999 (1992).

[44] B. Cichocki and K. Hinsen, Physica A 166, 473 (1990); 187, 133 (1992).

[45] W. C. K. Poon (unpublished).

[46] A. Imhof, A. van Blaaderen, G. Maret, J. Mellema, and J. K. G. Dhont, J. Chem. Phys. 100, 2170 (1994).

[47] Diffusion and Heat Flow in Liquids, edited by H. J. V. Tyrell (Butterworth, London, 1961).

[48] A. van Blaaren, J. Peetermans, G. Maret, and J. K. Dhont, J. Chem. Phys. 96, 4591 (1992).

[49] D. Chatenay, W. Urbach, R. Messager, and D. Langevin, J. Chem. Phys. 86, 2343 (1986).

[50] R. Zwanzig, J. Chem. Phys. 79, 4507 (1983). 